\title{
On the triangle space of a random graph*
}

\author{
B. Demarco, A. Hamm and J. Kahn
}

\begin{abstract}
Settling a first case of a conjecture of M. Kahle on the homology of the clique complex of the random graph $G=G_{n, p}$, we show, roughly speaking, that (with high probability) the triangles of $G$ span its cycle space whenever each of its edges lies in a triangle (which happens (w.h.p.) when $p$ is at least about $\sqrt{(3 / 2) \ln n / n}$, and not below this unless $p$ is very small). We give two related proofs of this statement, together with a fundamental "stability" theorem for triangle-free subgraphs of $G_{n, p}$, originally due to Kohayakawa, Łuczak and Rödl, that underlies the first of our proofs.
\end{abstract}

AMS 2010 SUBJECT Classifications: 05C80, 05C35, 05D40, 55U10, $60 \mathrm{C} 05$.

KEYWORDS AND PHRASES: Random graph, Kahle's conjecture, homology of the clique complex, threshold, stability theorem.

\section{Introduction}

The primary purpose of this paper is to prove a first case (Theorem 1.2) of a conjecture of M. Kahle on the homology of the clique complex of the (usual) random graph $G_{n, p}$. We will give two (not unrelated) proofs of this. Underlying the first is Theorem 1.4, a (known) "stability" theorem for triangle-free subgraphs of $G_{n, p}$, and our second main contribution is an alternative proof of this basic result. We begin with some background.

All graphs will have the vertex set $V=[n]=\{1 \ldots n\}$, so we will often fail to distinguish between a graph $G$ and its edge set and will tend to regard subgraphs of $G$ as subsets of $E(G)$. Recall that a cut of $G$ is $\nabla(W, V \backslash W)$, the set of edges of $G$ joining $W$ and $V \backslash W$ for some $W \subseteq V$.

More or less following [7], we set, for a given $G, \mathcal{E}=\mathcal{E}(G)=(\mathbf{Z} / 2)^{E(G)}$ (the edge space of $G$ ). We regard elements of $\mathcal{E}$ as subgraphs of $G$ in the natural way (namely, identifying a subgraph with its indicator), and write "+" for symmetric difference. The cycle space, $\mathcal{C}=\mathcal{C}(G)$ is the subspace of $\mathcal{E}$ spanned by the cycles, and $\mathcal{C}^{\perp}(:=\{H:\langle F, H\rangle=0 \forall F \in \mathcal{C}\}$ with the usual inner product) is precisely the set of cuts (which, note, includes $\emptyset$ ). We are

\footnotetext{
${ }^{*}$ Supported by NSF grant DMS0701175.
} 
particularly interested in the triangle space, $\mathcal{T}=\mathcal{T}(G)$, the subspace of $\mathcal{C}$ spanned by the triangles of $G$. Recall (see e.g. [24]) that the clique complex, $X(G)$, of a graph $G$ is the simplicial complex whose faces are the (vertex sets of) cliques of $G$.

In the rest of this section we write $G$ for $G_{n, p}$, where, as usual, $p=p(n)$. A precise possibility, suggested by M. Kahle ([18]; see also [16, 17]) and proved by him for $\Gamma=\mathbf{Q}[17]$, is

Conjecture 1.1. Let $\Gamma$ be either $\mathbf{Z}$ or a field. For each positive integer $k$ and $\varepsilon>0$, if

$$
p>(1+\varepsilon)[(1+k / 2)(\log n / n)]^{1 /(k+1)},
$$

then w.h.p. $H_{k}(X(G), \Gamma)=0$, where $H_{k}$ denotes kth homology group.

(Here log means ln and an event holds with high probability (w.h.p.) if its probability tends to 1 as $n \rightarrow \infty$.) We omit topological definitions, since we will not need them in what follows; see for example $[24,16]$. For $k=0$ with, of course, $H$ replaced by the reduced homology $\tilde{H}$-Conjecture 1.1 is more or less the classical result of Erdös and Rényi [9] giving the threshold for connectivity of $G_{n, p}$.

We will prove Conjecture 1.1 for $k=1$ and $\Gamma=\mathbf{Z} / 2$, which, being the first unsettled case, has apparently been the subject of some previous efforts $[1,18]$. Note that here the conclusion $\left(H_{1}(X(G), \mathbf{Z} / 2)=0\right)$ is just $\mathcal{T}(G)=\mathcal{C}(G)$, so that the desired statement is

Theorem 1.2. If $C>\sqrt{3 / 2}$ is fixed and $p>C \sqrt{\log n / n}$, then w.h.p. $\mathcal{T}(G)=\mathcal{C}(G)$.

We will actually prove the following more precise version, in which we set $Q=\{$ every edge is in a triangle .

\section{Theorem 1.3.}

$$
\max _{p} \operatorname{Pr}(G \text { satisfies } Q \text { and } \mathcal{T}(G) \neq \mathcal{C}(G)) \rightarrow 0 \quad(n \rightarrow \infty)
$$

This gives Theorem 1.2, since it is easy to see (see (16)) that for $p$ as in that theorem, w.h.p. every edge of $G$ does lie in a triangle. Note also (see Proposition 2.7) that for significantly smaller $p, Q$ is unlikely; so Theorem 1.3 is really about $p$ roughly as in Theorem 1.2 .

It seems likely that Theorem 1.3 can be strengthened to say that if $e_{1} \ldots e_{m}\left(m=\left(\begin{array}{c}n \\ 2\end{array}\right)\right)$ is a random (uniform) ordering of $\left(\begin{array}{c}{[n]} \\ 2\end{array}\right)$ and $G_{i}$ is the graph on $[n]$ with edge set $\left\{e_{1} \ldots e_{i}\right\}$, then w.h.p. there is no $i$ for which $G_{i}$ 
satisfies $Q$ and $\mathcal{T}\left(G_{i}\right) \neq \mathcal{C}\left(G_{i}\right)$. We have not really thought about whether proving this would require more than a small extension of the present argument.

As mentioned above, we will also give a new proof of

Theorem 1.4. For each $\eta>0$ there is a $C$ such that if $p>C n^{-1 / 2}$, then w.h.p. each triangle-free subgraph of $G$ of size at least $|G| / 2$ can be made bipartite by deletion of at most $\eta n^{2} p$ edges.

This seminal result — essentially Theorem 8.34 of [14] — seems due to Kohayakawa, Łuczak and Rödl [21], though Tomasz Łuczak [23] tells us it was already known (within some small circle) at the time. (Theorem 1.4 is a slightly restricted version of the actual result, corresponding to what is in [14]; see Theorem 8.1 below for the full statement.)

Theorem 1.4 is a "stability" version of the following "density" theorem, which is essentially due to Frankl and Rödl [10]. (More precisely, this is a little stronger than what is stated in [10], but is easily gotten from their proof; see also [12] or [14, Theorem 8.14].) Write $t(H)$ for the maximum size of a triangle-free subgraph of $H$.

Theorem 1.5. For each $\gamma>0$ there is a $C$ such that if $p>C n^{-1 / 2}$, then w.h.p. $t(G)<(1+\gamma)|G| / 2$.

The relation between Theorems 1.5 and 1.4 is like that between Turán's Theorem [31] and the Erdös-Simonovits "stability theorem" [29], which says, roughly, that any $K_{r}$-free graph with about $(1-1 /(r-1))\left(\begin{array}{l}n \\ 2\end{array}\right)$ edges is nearly $(r-1)$-partite. The extension of Theorem 1.5 to larger $r$, conjectured in [21], was proved by Conlon and Gowers [4] and Schacht [28]; the corresponding extension of Theorem 1.4, suggested in [19, 21], was also proved in [4], then again in [26] (building on [28]), and very recently in [3] and [27]. (All of these papers treat more general forbidden subgraphs.)

The original proof of Theorem 1.4 (see [21, 14]) uses a sparse version of Szemerédi's Regularity Lemma [30] due to Kohayakawa [19] and Rödl (unpublished; see [19]), together with the triangle case of the "KŁR Conjecture" of [21] (which has recently been proved in full by Balogh et al. [3]), while the ingenious recent proofs avoid such tools (apart from a superficial use of the "graph removal lemma" in connection with the present Lemma 3.3; see the remark following the statement of that lemma).

Our (unbiased) feeling is that the argument given here is the simplest to date, even compared to specializations of earlier approaches to the single case covered by Theorem 1.4 (though Jozsi Balogh [2] tells us that the specialization of [3] is also reasonably simple); it is also of a somewhat different 
flavor than earlier work, though there are similarities. All proofs depend on versions of Lemma 3.3. The argument given here also has in common with [3] and [27] the use of a small subset of a possible violator $F$ to significantly restrict the universe from which the remainder of $F$ must be drawn; but the mechanism by which we accomplish this is (in a word) more "dynamic": it is based on sampling from $G$, while [3] and [27] depend on an a priori description of (all) triangle-free subgraphs of $K_{n}$. The crucial (simple) point supporting our version is Lemma 3.2, which seems interesting in itself.

The present proof was obtained independently of $[3,27]$ and was part of the first author's Ph.D. thesis, which was defended around the time [3] and [27] were posted [5]. At this writing we do not know whether the approach can be extended to prove some of the more general results mentioned above; generalizing to $K_{r}$ would just require the corresponding extension of Lemma 3.2, which seems true though we do not yet see a proof.

An interest in reproving Theorem 1.4-partly motivated by an application of that theorem in [6] — was actually the starting point for the present work, as follows. It is not too hard to show that, roughly speaking, if $p$ is as in Theorem 1.4, then w.h.p. every triangle-free $F \subseteq G$ with $|F| \geq|G| / 2$ has even intersection with most triangles of $G$. (This again is essentially from [10], following an idea of Goodman [11]; see also [14, Sec. 8.2].) So in thinking about a new proof of Theorem 1.4, we wondered whether some insight might be gained by understanding what happens when one replaces "most" by "all." This led to the question addressed in Theorem 1.2, which we realized only later was a known problem.

The rest of the paper is organized as follows. Section 2 consists of various standardish preliminaries, while Section 3 contains statements of more interesting lemmas, which are then proved in Sections 4-6. Section 7 gives the easy derivation of Theorem 1.3 from Theorem 1.4 and Lemma 3.1, and our proof of Theorem 1.4 is given in Section 8. Finally, Section 9 contains a sketch of a separate proof of Theorem 1.3 that avoids Theorem 1.4 (this is put off until the end of the paper to allow reference to Sections 7 and 8).

Usage As noted above, all our graphs will have vertex set $V=[n]$. We use $v \ldots z$ for vertices, often without explicitly specifying, e.g., " $x \in V$," and $x y$ for the edge more properly written $\{x, y\}$. We use $|H|=|E(H)|$ (the size of $H), N_{H}(x)=\{y: x y \in H\}$ (the neighborhood of $x$ in $H$ ), $d_{H}(x)=\left|N_{H}(x)\right|$ (the degree of $x$ in $H$ ) and $d_{H}(x, y)=\left|N_{H}(x) \cap N_{H}(y)\right|$. For disjoint $S, T \subseteq V$, $\nabla_{H}(S, T)$ is the set of edges joining $S, T$ in $H, \nabla_{H}(S)$ is $\nabla_{H}(S, V \backslash S)$-as noted earlier such a set of edges, for which we will often write simply $\Pi$, is 
a cut of $H$-and $\nabla_{H}(v)=\nabla_{H}(\{v\})$. As usual, $H[S]$ is the subgraph of $H$ induced by $S$. We use $T(H)$ for the set of triangles of $H$.

In much of the paper we will take $G=G_{n, p}$ and use this as the default for $H$, so that (e.g.) $N(x)=N_{G}(x), d(x)=d_{G}(x), \nabla(S, T)=\nabla_{G}(S, T)$ and, for $B \subseteq V, N_{B}(x)=N(x) \cap B$.

Finally, we use log for $\ln , B(m, p)$ for a random variable with the binomial distribution $\operatorname{Bin}(m, p)$, and " $a=(1 \pm \vartheta) b$ " for " $(1-\vartheta) b \leq a \leq(1+\vartheta) b$."

\section{Preliminaries}

Here we record some routine probabilistic basics. We use "Chernoff's inequality" in the following form (see [14, Theorem 2.1]), where for $x \geq-1$, $\varphi(x)=(1+x) \log (1+x)-x$.

Theorem 2.1. For $\xi=B(n, p), \mu=n p$ and any $\lambda \geq 0$,

$$
\operatorname{Pr}(\xi \geq \mu+\lambda) \leq \exp \left[-\frac{\lambda^{2}}{2(\mu+\lambda / 3)}\right]
$$

and

$$
\operatorname{Pr}(\xi \leq \mu-\lambda) \leq \exp [-\mu \varphi(-\lambda / \mu)] \leq \exp \left[-\frac{\lambda^{2}}{2 \mu}\right] .
$$

(We will not need the more precise version of the first bound.)

We will also (in Section 8) need the following Azuma-Hoeffding type bound. (A similar statement can be extracted from, e.g., the discussion in Section 3 of [15] (see (33) and Lemma 3.9(a)); but we include the simple proof.)

Lemma 2.2. Let $X=X\left(\xi_{1} \ldots \xi_{m}\right)$ where the $\xi$ 's are i.i.d., each with the distribution $\operatorname{Ber}(q)$, and suppose $X$ is Lipschitz (that is, changing the value of a single $\xi_{i}$ changes the value of $X$ by at most 1$)$. Then for any $t \in[0,2 m q]$, each of $\operatorname{Pr}(X-\mathrm{E} X<-t)$ and $\operatorname{Pr}(X-\mathrm{E} X>t)$ is at most $\exp \left[-t^{2} /(4 m q)\right]$.

Proof. We first observe that if the r.v. $W$ with $\mathrm{E} W=0$ satisfies $\operatorname{Pr}(W=$ $a)=q=1-\operatorname{Pr}(W=b)$ for some $a, b$ with $|a-b| \leq 1$, then for any $\zeta \in[0,1]$,

$$
\mathrm{E} e^{\zeta W} \leq e^{-\zeta q}\left[1-q+q e^{\zeta}\right] \leq e^{\zeta^{2} q},
$$

where the first inequality follows from the convexity of $e^{x}$ and the second is an easy Taylor series calculation.

Set $X_{i}=\mathrm{E}\left[X \mid e_{1} \ldots e_{i}\right], Z_{i}=X_{i}-X_{i-1}(i \in[m])$ and $Z=\sum Z_{i}$. Then

$$
\operatorname{Pr}(X-\mathrm{E} X>t)=\operatorname{Pr}\left(e^{\zeta Z}>e^{\zeta t}\right)<e^{-\zeta t} \mathrm{E} e^{\zeta Z},
$$


while (1) and induction on $m$ (used in (3) and (4) respectively) give, again for $\zeta \in[0,1]$,

$$
\begin{aligned}
\mathrm{E} e^{\zeta Z}=\mathrm{E} e^{\zeta\left(Z_{1}+\cdots+Z_{m}\right)} & =\mathrm{E}\left[\mathrm{E}\left(e^{\zeta\left(Z_{1}+\cdots+Z_{m}\right)} \mid \xi_{1} \ldots \xi_{m-1}\right)\right] \\
& =\mathrm{E}\left[e^{\zeta\left(Z_{1}+\cdots+Z_{m-1}\right)} \mathrm{E}\left(e^{\zeta Z_{m}} \mid \xi_{1} \ldots \xi_{m-1}\right)\right] \\
& \leq \mathrm{E}\left[e^{\zeta\left(Z_{1}+\cdots+Z_{m-1}\right)} e^{\zeta^{2} q}\right] \\
& \leq e^{\zeta^{2} m q}
\end{aligned}
$$

Finally, inserting this in $(2)$ and taking $\zeta=t /(2 m q)$ gives the desired bound.

For the rest of this section we set $G=G_{n, p}$, and assume $p$ is at least $n^{-1 / 2}$. Of course, many of the statements below hold in more generality, but there seems no point in worrying about this. All proofs are quite straightforward, so we give only one or two representative arguments.

Proposition 2.3. W.h.p.

$$
|G|=(1 \pm o(1)) n^{2} p / 2
$$

and

$$
d(x)=(1 \pm o(1)) n p \quad \forall x .
$$

If $p>n^{-1 / 2} \log ^{1 / 2} n$, then w.h.p.

$$
d(x, y)<4 n p^{2} \quad \forall x, y .
$$

Proposition 2.4. (a) For each $\delta$ there is a $K$ such that w.h.p.

$$
|\nabla(S, T)|=(1 \pm \delta)|S||T| p
$$

for all disjoint $S, T \subseteq V$ of size at least $K p^{-1} \log n$.

(b) For each fixed $\delta>0$, w.h.p.

$$
|\nabla(S)|=(1 \pm \delta)|S|(n-|S|) p \quad \forall S \subseteq V .
$$

For $X, Y$ (not necessarily disjoint) subsets of $V$, set $\zeta(X, Y)=\zeta_{G}(X, Y)=$ $|\{(x, y) \in X \times Y: x y \in G\}|$.

Proposition 2.5. For any $\varepsilon>0$ w.h.p.

$$
\zeta(Y, Z)=(1 \pm \varepsilon)|Y||Z| p
$$

for all $Y, Z \subseteq V$ with $|Y||Z|>8 \varepsilon^{-2} p^{-1} n$. 
Proof (sketch). We may assume $\varepsilon$ is small. It is easy to see that for a given $Y, Z, \zeta(Y, Z)$ can be written as $B\left(m_{1}, p\right)+B\left(m_{2}, p\right)$ with $m_{1}+m_{2}=|Y||Z|-$ $|Y \cap Z|$. Failure of (10) (for $Y, Z$ ) then requires that at least one of these binomials differ from its mean by at least (essentially) $\varepsilon|Y||Z| p / 2$, and the probability of each of these events is bounded by $\exp \left[-\varepsilon^{2}|Y||Z| p /[8(1+\varepsilon / 3)]\right.$, which is $o\left(2^{-n}\right)$ for $Y, Z$ as in the proposition.

Proposition 2.6. (a) There is a $K$ such that w.h.p. for all $v, S \subseteq N(v)$ and $T=N(v) \backslash S$,

$$
|| \nabla(S, T)|-| S|| T|p|<K n^{3 / 2} p^{2}
$$

and

$$
|G[S]|< \begin{cases}|S|^{2} p / 2+K n^{3 / 2} p^{2} & \text { in general } \\ o\left(|S| n p^{2}\right) & \text { if }|S|=o(n p)\end{cases}
$$

(b) There is an $\alpha>0$ such that if $p>1.2 \sqrt{\log n / n}$ then w.h.p.

$$
|\nabla(S, T)|>\alpha|S| n p^{2}
$$

whenever $v \in V, S \subseteq N(v), T=N(v) \backslash S$ and $2 \leq|S| \leq|T|$.

(c) There is a $K$ so that w.h.p. for all $v$ and $S, T$ disjoint subsets of $N(v)$ with $|T|>n p / 3$ and $|S|>K / p$,

$$
|\nabla(S, T)|>0.9|S||T| p
$$

Remark. The 1.2 in (b) is just a convenient choice between 1 and $\sqrt{3 / 2}$.

Proof (sketch). In each case, by Proposition 2.3 (see (6)), it is enough to bound the probability that the assertion fails at some $v$ with $d(v)=(1 \pm$ $o(1)) n p$. We use $s$ and $t$ for $|S|$ and $|T|$. Having chosen $v$ and $N(v)$ of size $m=(1 \pm o(1)) n p$, we may bound the number of possibilities for $(S, T)$ (with given $s, t)$ by $\left(\begin{array}{c}m \\ s\end{array}\right)$ in (a),(b) and (say) $\left(\begin{array}{c}m \\ s\end{array}\right)\left(\begin{array}{c}m \\ t\end{array}\right)$ in (c). On the other hand, once we have specified $S$ (and $T$ if we are in (c)), we are just bounding a deviation probability for some binomial random variable, and the required bounds can (with a little effort) be read off from Theorem 2.1.

For example, the most delicate of these assertions is (b) (which is most delicate for $s=2$ ). In general for (b) with $s=o(n p)$ (which is more than is needed from (b), since (a) covers $s$ above about $\sqrt{n}$ ), we may, using 
Theorem 2.1, bound the probability of a violation with $|S|=s$ by

$$
\begin{aligned}
& n\left(\begin{array}{c}
(1+o(1)) n p \\
s
\end{array}\right) \exp \left[-(1-o(1)) s n^{2} p \varphi(-1+\alpha+o(1))\right] \\
& \quad<n\left\{n p \exp \left[-(1-\beta) n p^{2}\right]\right\}^{s}
\end{aligned}
$$

where $\beta=\beta_{\alpha} \rightarrow 0$ as $\alpha \rightarrow 0$. (The initial $n$ is for the choice of $v$, and we have used $s=o(n p)$ and $d(v)=(1 \pm o(1)) n p$ to say $t=(1-o(1)) n p$. ) Now $n p \exp \left[-(1-\beta) n p^{2}\right]$ is decreasing in $p$, so is at most $1.2 \sqrt{\log n} n^{1 / 2-(1-\beta) 1.44}$ for $p$ as in (b). Thus, for slightly small $\alpha$, the sum over $s \geq 2$ of the right hand side of (15) is bounded by some fixed negative power of $n$.

Finally we should justify the two comments following the statement of Theorem 1.3, namely that the property $Q$ (every edge of $G$ is in a triangle) holds w.h.p. if $p$ is as in Theorem 1.2 and fails w.h.p. if $p$ is significantly smaller. The first of these is trivial: if $X$ is the number of edges of $G$ not lying in triangles, then

$$
\mu(p):=\mathrm{E} X=\left(\begin{array}{c}
n \\
2
\end{array}\right) p\left(1-p^{2}\right)^{n-2},
$$

which is $o(1)$ for $p>\sqrt{(3 / 2+\varepsilon) \log n / n}$ (where, here and in the following proposition, $\varepsilon$ is any positive constant). The second assertion is just a second moment method calculation, whose outcome we record as

Proposition 2.7. If $\mu(p)=\omega(1)$, then $\operatorname{Pr}(X=0)=o(1)$ (where $X$ and $\mu(p)$ are as in (16)); in particular, this is true if $p<\sqrt{(3 / 2-\varepsilon) \log n / n}$ with $\varepsilon$ a positive constant.

Proof. We have $X=\sum \mathrm{E} A_{x y}$ with the sum over edges $x y$ of $K_{n}$ and $A_{x y}$ the indicator of $\{x y \in G$ and $x y$ lies in no triangle of $G\}$. We then observe that for $x, y, z, w$ distinct,

$$
\mathrm{E} A_{x y} A_{z w}<p^{2}\left(1-p^{2}\right)^{2(n-4)} \text { and } \mathrm{E} A_{x y} A_{x z}<p^{2}\left(1-2 p^{2}+p^{3}\right)^{n-3},
$$

which with (16) (and minor calculations which we omit) gives $\operatorname{Var}(\mathrm{X}) / \mathrm{E}^{2} X=$ $O(1 / \mu(p))$.

\section{Main lemmas}

We collect here a few main points underlying the proofs of Theorems 1.3 and 1.4. As earlier we write $G$ for $G_{n, p}$.

Theorem 1.3 says that (for any $p$ ) it is unlikely that $Q$ holds but $\mathcal{T}(G) \neq$ $\mathcal{C}(G)$ (or, equivalently, $\mathcal{T}(G)^{\perp} \neq \mathcal{C}(G)^{\perp}$ ). As shown in Section 7, this follows 
easily from Theorem 1.4 once we have ruled out "small" members of $\mathcal{T}^{\perp}(G) \backslash$ $\mathcal{C}^{\perp}(G)$ :

Lemma 3.1. For $Q$ as in Theorem 1.3 and fixed $\eta>0$,

$$
\max _{p} \operatorname{Pr}\left(Q \wedge\left[\exists F \in \mathcal{T}^{\perp}(G) \backslash \mathcal{C}^{\perp}(G),|F|<(1-\eta) n^{2} p / 4\right]\right)<o(1) .
$$

For a graph $H$ on $[n]$ and $K \subseteq H$, set

$B(K, H)=\left\{e \in K_{n} \backslash H\right.$ : there is no triangle $\{e, f, g\}$ with $\left.f, g \in K\right\}$.

In the proof of Theorem 1.4 we will choose $G$ by first choosing a subgraph $G_{0} \sim G_{n, \vartheta p}$ and then placing edges of $K_{n} \backslash G_{0}$ in $G$ with probability (1 $\vartheta) p /(1-\vartheta p)$ (independently). Then specification of $F_{0}=F \cap G_{0}$, for a triangle-free $F \subseteq G$, limits the possibilities for $F \backslash G_{0}$ to subsets of $B\left(F_{0}, G_{0}\right)$, and we will want to say this set is small; such an assertion is supported by the next lemma (which we will apply with $G, F$ and $p$ replaced by $G_{0}, F_{0}$ and $\vartheta p)$.

Lemma 3.2. For each $\delta>0$ there are $C$ and $\varepsilon>0$ such that if $p>$ $C n^{-1 / 2}$ then w.h.p. $|B(F, G)|<(1+\delta) n^{2} / 4$ for each $F \subseteq G$ of size at least $(1-\varepsilon) n^{2} p / 4$.

Finally, we need the following simple deterministic fact, in which we write $\tau(F)$ for the number of triangles in $F$.

Lemma 3.3. If $F \subseteq K_{n}$ satisfies $|F|>(1-\delta) n^{2} / 4$ and $|F \backslash \Pi|>\eta n^{2}$ for every cut $\Pi$, then $\tau(F)>\frac{1}{12}(\eta-3 \delta-o(1)) n^{3}$.

Remark. As suggested earlier this is not really new, versions of a much more general statement having been used in [4, 26, 3, 27]; nonetheless we include the simple proof (see Section 4 ), both to make our argument self-contained and to give a reasonable dependence of $C$ on $\eta$ in Theorem 1.4. The results corresponding to Lemma 3.3 in $[4,26,3,27]$ are proved-presumably just for convenience - using the "graph removal lemma" of [8] (so for Lemma 3.3 itself the original "triangle removal lemma" of Ruzsa and Szemerédi [25]), which for example gives Lemma 3.3 with both $\delta$ and $\frac{1}{12}(\eta-3 \delta-o(1))$ replaced by some tiny constant depending on $\eta$.

\section{Proof of Lemma 3.1}

We need one easy preliminary observation, which will show up again in the proof of Theorem 1.3. 
Proposition 4.1. Let $G$ be a graph and $F \subseteq G$, and suppose $F^{\prime}, F^{\prime \prime}$ are (respectively) minimum and maximum size members of $F+\mathcal{C}^{\perp}(G)$. Then

$$
\forall v \quad d_{F^{\prime}}(v) \leq d_{G \backslash F^{\prime}}(v) \quad \text { and } \quad d_{F^{\prime \prime}}(v) \geq d_{G \backslash F^{\prime \prime}}(v) .
$$

(For example if $F^{\prime}$ violates the first condition (at $v$ ), then $F^{\prime}+\nabla(v) \in$ $F+\mathcal{C}^{\perp}(G)$ is smaller than $F^{\prime}$.)

We turn to the proof of Lemma 3.1, noting that, by Proposition 2.7, it is enough to bound the probability in (17) when (say) $p>1.2 \sqrt{\log n / n}$, and for this it is enough to show that the event in (17) - that is,

$$
Q \wedge\left[\exists F \in \mathcal{T}^{\perp}(G) \backslash \mathcal{C}^{\perp}(G),|F|<(1-\eta) n^{2} p / 4\right]
$$

- cannot occur if $G$ satisfies the conclusions of Propositions 2.3, 2.4 and 2.6. Suppose instead that these conclusions are satisfied and (18) holds, and let $F$ be a smallest member of $T^{\perp}(G) \backslash \mathcal{C}^{\perp}(G)$ and $J=G \backslash F$. By Proposition 4.1 we have

$$
d_{J}(v) \geq d_{F}(v) \quad \forall v
$$

For disjoint $S, T \subseteq V$, set $\Psi(S, T)=|\nabla(S, T)|-2|G[S]|$. Since

$$
\sum_{v}\left|\nabla\left(N_{F}(v), N_{J}(v)\right)\right|=2|\{T \in T(G):|F \cap T|=2\}|=2 \sum_{v}\left|G\left[N_{F}(v)\right]\right|,
$$

we have

$$
\sum_{v} \Psi\left(N_{F}(v), N_{J}(v)\right)=0
$$

Let $\varepsilon=\eta / 2$ and set $V_{1}=\left\{v: d_{F}(v)>(1-\varepsilon) n p / 2\right\}, V_{2}=\left\{v \in V \backslash V_{1}\right.$ : $\left.d_{F}(v) \geq 2\right\}$ and $V_{3}=V \backslash\left(V_{1} \cup V_{2}\right)$. Note that $Q$ (with $F \neq \emptyset$, which is all we are now using from $\left.F \notin \mathcal{C}^{\perp}\right)$ implies $V_{1} \cup V_{2} \neq \emptyset$. The conclusions of parts (a) and (b) of Proposition 2.6 give, for some fixed positive $\delta$ and $L$,

$$
\begin{aligned}
\sum_{v} \Psi\left(N_{F}(v), N_{J}(v)\right) & \geq \delta \sum_{v \in V_{2}} d_{F}(v) n p^{2}-L\left|V_{1}\right| n^{3 / 2} p^{2} \\
& =n p^{2}\left[\delta \sum_{v \in V_{2}} d_{F}(v)-L\left|V_{1}\right| n^{1 / 2}\right]
\end{aligned}
$$

(For $v \in V_{1},(11)$ and (12) give

$$
\Psi\left(N_{F}(v), N_{J}(v)\right)>\left(d_{F}(v) d_{J}(v)-d_{F}^{2}(v)\right) p-3 K n^{3 / 2} p^{2} \geq-3 K n^{3 / 2} p^{2} .
$$


A similar discussion gives $\Psi\left(N_{F}(v), N_{J}(v)\right)>\delta d_{F}(v) n p^{2}$ for $v \in V_{2}$, where for smaller $d_{F}(v)$ we use (13) and the second bound in (12).)

On the other hand, we will show that

$$
\sum_{v \in V_{2}} d_{F}(v)=\omega\left(\left|V_{1}\right| n^{1 / 2}\right),
$$

which (with (21)) contradicts (20) and completes the proof.

We first observe that (6), (14) and (19) imply that for every $v \in V_{1}$,

(23) $\left|\left\{w \in N(v): \min \left\{\left|N(w) \cap N_{F}(v)\right|,\left|N(w) \cap N_{J}(v)\right|\right\}<\frac{n p^{2}}{4}\right\}\right|<o(n p)$,

so in particular

$$
\left|N(v) \cap V_{3}\right|=o(n p) .
$$

(If $z \in N(v) \cap V_{3}$, then either $z \in N_{F}(v)$, whence $\nabla\left(z, N_{J}(v)\right) \subseteq F$ and (by the definition of $\left.V_{3}\right) N(z) \cap N_{J}(v)=\emptyset$, or, similarly, $z \in N_{J}(v)$ and $\left|N(z) \cap N_{F}(v)\right| \leq 1$.)

Now $|F|<(1-\eta) n^{2} p / 4$ implies $\left|V_{1}\right|<(1-\varepsilon) n$ (since $(1-\eta) n^{2} p / 4>$ $|F|>(1 / 2)\left|V_{1}\right|(1-\varepsilon) n p / 2$ implies $\left.\left|V_{1}\right|<(1-\eta) n /(1-\varepsilon)<(1-\varepsilon) n\right)$. So by (9) we have

$$
\left|\nabla\left(V_{1}\right)\right|>(1-o(1))\left|V_{1}\right| \varepsilon n p,
$$

which in view of $(24)$ gives

$$
\left|\nabla\left(V_{1}, V_{2}\right)\right|>(1-o(1))\left|V_{1}\right| \varepsilon n p .
$$

On the other hand, we may assume $\left|\nabla_{F}\left(V_{1}, V_{2}\right)\right|=o\left(\left|V_{1}\right| n p\right)$ (or we have (22)), which gives at least $(1-o(1))\left|V_{1}\right| \varepsilon n p$ pairs $(v, w)$ with

$$
v \in V_{1}, w \in V_{2}, v w \in J \text { and }\left|N_{F}(w) \cap N_{F}(v)\right|>n p^{2} / 4
$$

(since by (23) only $o\left(\left|V_{1}\right| n p\right)$ pairs satisfying the first three conditions are eliminated by the last). This gives $\Omega\left(\left|V_{1}\right| n p \cdot n p^{2}\right)$ triples $(v, w, z)$ with $v \in V_{1}$, $w \in V_{2}, v w \in J$ and $z \in\left|N_{F}(w) \cap N_{F}(v)\right|$. But since each $(w, z)$ belongs to at most $4 n p^{2}$ such triples (see (7)), this says that there are at least $\Omega\left(\left|V_{1}\right| n p\right)$ edges of $F$ meeting $V_{2}$, so we have (22). 


\section{Proof of Lemma 3.2}

We prove the lemma with $\varepsilon=.05 \delta$ and $C=4 \varepsilon^{-2}$. For $F \subseteq G$, set $L(F, G)=$ $\left\{x y \in E\left(K_{n}\right): d_{F}(x, y) \neq 0\right\}$. It is enough to show that for suitable $C$ and $\varepsilon$, and $p$ as in Lemma 3.2, w.h.p.

$$
|L(F, G)|>(1-\delta) n^{2} / 4
$$

for each $F \subseteq G$ of size at least $(1-\varepsilon) n^{2} p / 4$. In fact, all this needs from the randomization is the property

$$
\zeta(Y, Z)=(1 \pm \varepsilon)|Y||Z| p \quad \forall Y, Z \subseteq V \text { with }|Y| \geq \varepsilon n p \text { and }|Z| \geq \varepsilon n / 2
$$

which according to Proposition 2.5 holds w.h.p.; thus, we assume (28) holds in $G$ and proceed deterministically.

Given $F \subseteq G$, set $L=L(F, G)$ and, for $x \in V$,

$$
\zeta(x)=\zeta_{G}\left(N_{F}(x), N_{L}(x)\right) \quad(=|\{(y, z): x y \in F, x z \in L, y z \in G\}|) .
$$

Then

$$
\zeta(x) \geq \zeta_{F}\left(\left(N_{F}(x), N_{L}(x)\right)=\sum_{y \in N_{F}(x)}\left(d_{F}(y)-1\right)\right.
$$

Heading for a companion upper bound, we say $x$ is $\operatorname{good}($ for $F$ ) if

$$
\left|\left\{y \in N_{F}(x): d_{F}(y)>\varepsilon n p\right\}\right|>\varepsilon n p
$$

(and bad otherwise), and let $F^{*}=\{x y \in F: x, y$ are good $\}$. We need a few little observations. First (we assert)

$$
\left|F \backslash F^{*}\right| \leq 2 \varepsilon n^{2} p .
$$

To see this, just notice that an edge of $F \backslash F^{*}$ either contains a vertex of $F$-degree at most $\varepsilon n p$ or, for some bad $x$, is one of at most $\varepsilon n p$ edges of $F$ at $x$ that do not contain a vertex of $F$-degree at least $\varepsilon n p$.

Second, notice that

$$
x \text { good } \Rightarrow d_{L}(x)>\varepsilon n / 2 .
$$

For if this fails, then there are $Y, Z \subseteq V$ (namely $Y=N_{F}(x), Z=N_{L}(x)$ ) with $|Y|>\varepsilon n p,|Z| \leq \varepsilon n / 2$ and $\zeta(Y, Z) \geq|Y| \varepsilon n p$, which implies a violation of (28) (at $Y$ and some ( $\varepsilon n / 2)$-superset of $Z$ ). 
Third, again using (28), we find that if $x$ is good (or if just $d_{F}(x)>\varepsilon n p$ and the conclusion of (31) holds) then

$$
\zeta(x)<(1+\varepsilon) d_{F}(x) d_{L}(x) p,
$$

which with (29) gives (for good $x$ )

$$
\begin{aligned}
d_{L}(x) & >\left[(1+\varepsilon) p d_{F}(x)\right]^{-1} \sum_{y \in N_{F}(x)}\left(d_{F}(y)-1\right) \\
& >\frac{1-\varepsilon}{p d_{F}(x)} \sum_{y \in N_{F}(x)} d_{F}(y)
\end{aligned}
$$

where, since $x$ is good (and $p$ is large), passing from $(1+\varepsilon)^{-1}$ to $1-\varepsilon$ takes care of the missing " -1 " in the second line.

But then (using (30) and our lower bound on $|F|$ in the last line)

$$
\begin{aligned}
2|L| & \geq \sum_{x \text { good }} d_{L}(x)>\frac{1-\varepsilon}{p} \sum_{x \text { good }} \sum_{y \in N_{F}(x)} \frac{d_{F}(y)}{d_{F}(x)} \\
& \geq \frac{1-\varepsilon}{p} \sum_{x y \in F^{*}}\left[\frac{d_{F}(y)}{d_{F}(x)}+\frac{d_{F}(x)}{d_{F}(y)}\right] \\
& \geq 2(1-\varepsilon)\left|F^{*}\right| / p \\
& >2(1-\varepsilon)\left[(1-\varepsilon) n^{2} p / 4-2 \varepsilon n^{2} p\right] / p>(1-\delta) n^{2} / 2
\end{aligned}
$$

(so we have (27)).

\section{Proof of Lemma 3.3}

Suppose $F$ is as in the lemma and denote by $t_{i}$ the number of triangles of $K_{n}$ containing exactly $i$ edges of $F, i \in\{0,1,2,3\}$ (so $t_{3}=\tau(F)$ ). Writing $X$ for the number of pairs $(e, T)$ with $e \in F$ and $T$ a triangle of $K_{n}$ containing $e$, we have

$$
|F|(n-2)=X=t_{1}+2 t_{2}+3 t_{3}
$$

and, according to a nice observation of Goodman [11] (see [14, p. 209] for the easy proof),

$$
t_{1}+t_{2}<n^{3} / 8
$$


On the other hand,

$$
t_{1}+t_{3} \geq \eta n^{3} / 3
$$

since applying the hypothesized lower bound on the $|F \backslash \Pi|$ 's to the cuts $\Pi=\left(N_{F}(v), V \backslash N_{F}(v)\right)$ shows that each vertex lies in at least $\eta n^{2}$ of the triangles counted by $t_{1}+t_{3}$.

Now (32) and (33) (together with our assumption on $|F|$ ) imply

$$
\begin{aligned}
(1-\delta) n^{2}(n-2) / 4<|F|(n-2) & =t_{1}+2 t_{2}+3 t_{3} \\
& =2\left(t_{1}+t_{2}\right)-t_{1}+3 t_{3}<n^{3} / 4-t_{1}+3 t_{3},
\end{aligned}
$$

whence

$$
t_{1}-3 t_{3}<(\delta+o(1)) n^{3}
$$

and combining this with $(34)$ gives $t_{3}>\frac{1}{12}(\eta-3 \delta-o(1)) n^{3}$.

\section{Proof of Theorem 1.3}

By Proposition 2.7 and Lemma 3.1, it is enough to show that for $p>$ $1.2 \sqrt{\log n / n}$ and a fixed $\eta>0$, it is unlikely that $\mathcal{T}^{\perp}(G)$ contains an $F$ for which

$$
\min \left\{\left|F^{\prime}\right|: F^{\prime} \in F+\mathcal{C}^{\perp}(G)\right\}>(1-\eta) n^{2} p / 4 .
$$

Now if there is such an $F$, then by Proposition 4.1 there is one of size at least $|G| / 2$, and w.h.p. this also satisfies (say) $|F \backslash \nabla(A, B)|>0.1 n^{2} p$ for each partition $A \cup B$ of $V$; for, writing $\nabla$ for $\nabla(A, B)$, we have

$$
(1-\eta) n^{2} p / 4<|F+\nabla|=2|F \backslash \nabla|+|\nabla|-|F|<2|F \backslash \nabla|+o\left(n^{2} p\right),
$$

where we used Proposition 2.3 (to say $|G|>(1-o(1)) n^{2} p / 2$ ) and Proposition 2.4(a) (to say $\left.|\nabla|<(1+o(1)) n^{2} p / 4\right)$. But according to Theorem 1.4, the probability that there is such an $F$ is $o(1)$ even for $p>C n^{-1 / 2}$ (with $C$ as in Theorem 1.4).

\section{Proof of Theorem 1.4}

As mentioned in Section 1, we prove the slightly stronger version from [21]:

Theorem 8.1. For any $\eta>0$ there are $\varepsilon>0$ and $C$ such that if $p>C n^{-1 / 2}$ then w.h.p. each triangle-free subgraph of $G$ of size at least $(1-\varepsilon) n^{2} p / 4$ can be made bipartite by deletion of at most $\eta n^{2} p$ edges. 
Proof. As suggested in Section 3, we choose $G$ by first choosing a subgraph $G_{0} \sim G_{n, \vartheta p}$ and then placing edges of $K_{n} \backslash G_{0}$ in $G_{1}:=G \backslash G_{0}$ independently, each with probability $q:=(1-\vartheta) p /(1-\vartheta p)$.

Set $\vartheta=10^{-5} \eta^{2}$. According to Lemma 3.2 (and (5)), we may choose $\varepsilon<\vartheta$ and $C$ so that w.h.p.

$$
|G| \sim n^{2} p / 2, \quad\left|G_{0}\right| \sim n^{2} \vartheta p / 2
$$

and

$$
\left[F_{0} \subseteq G_{0},\left|F_{0}\right|>(1-2 \varepsilon)\left|G_{0}\right| / 2\right] \Rightarrow\left|B\left(F_{0}, G_{0}\right)\right|<(1+\vartheta) n^{2} / 4
$$

(with $B(\cdot, \cdot)$ as in Lemma 3.2). Let $Q$ be the event that (38) and (39) occur.

Call $F \subseteq G$ bad if it is triangle-free with $|F|>(1-\varepsilon) n^{2} p / 4$ and $|F \backslash \Pi|>$ $\eta n^{2} p$ for every $\Pi$. Let $R$ be the event that $G$ contains a bad $F$ and $S(\subseteq R)$ the event that some $F \subseteq G$ and $F_{0}=F \cap G_{0}$ satisfy

$$
F \text { is bad and }\left|F_{0}\right|>(1-2 \varepsilon) \vartheta n^{2} p / 4 .
$$

Then

$$
\operatorname{Pr}(S \mid R) \geq \operatorname{Pr}\left(B\left((1-\varepsilon) n^{2} p / 4, \vartheta\right)>(1-2 \varepsilon) \vartheta n^{2} p / 4\right)>1 / 2
$$

(e.g. by Theorem 2.1, which of course really gives $1-o(1)$ in place of $1 / 2)$; so we will have $\operatorname{Pr}(R)=o(1)$ (which is what we want) if we show $\operatorname{Pr}(S)=o(1)$, which, since $Q$ holds w.h.p., is the same as

$$
\operatorname{Pr}(Q \wedge S)=o(1) .
$$

Suppose then that $Q$ holds and that $F \subseteq G$ and $F_{0}:=F \cap G_{0}$ satisfy (40), and set $F_{1}=F \backslash F_{0}$ and $B=B\left(F_{0}, G_{0}\right)$. By (39) we have

$$
|B|<(1+\vartheta) n^{2} / 4 .
$$

Now according to Lemma $3.3, B$ must satisfy at least one of (say)

(i) $|B|<(1-0.1 \eta) n^{2} / 4$

(ii) there is a cut $\Pi$ for which $|B \backslash \Pi|<0.9 \eta n^{2}$;

(iii) $\tau(B)>.04 \eta n^{3}$.

On the other hand, since $F$ is bad (and $F_{1} \subseteq G \cap B$ ), we have:

$$
|G \cap B| \geq\left|F_{1}\right| \geq|F|-\left|G_{0}\right|>(1-3 \vartheta) n^{2} p / 4 ;
$$




$$
\begin{aligned}
|G \cap(B \backslash \Pi)| & =|(G \cap B) \backslash \Pi| \geq\left|F_{1} \backslash \Pi\right| \\
& \geq|F \backslash \Pi|-\left|G_{0}\right|>(\eta-\vartheta) n^{2} p
\end{aligned}
$$

for every cut $\Pi$ of $K_{n}$; and $X:=(G \cap B) \backslash F_{1}$ is a set of edges meeting (i.e. containing an edge of) each triangle of $G \cap B$, with

$$
|X|=|G \cap B|-\left|F_{1}\right|<|G \cap B|-(1-3 \vartheta) n^{2} p / 4
$$

Thus, if $Q \wedge S$ holds, then there is an $F_{0} \subseteq G_{0}$ such that $B=B\left(F_{0}, G_{0}\right)$ satisfies (42) and one of the following is true:

(a) $|B|<(1-0.1 \eta) n^{2} / 4$ and $|G \cap B|>(1-3 \vartheta) n^{2} p / 4$;

(b) there is a cut $\Pi$ for which

$$
|B \backslash \Pi|<0.9 \eta n^{2} \text { and }|G \cap(B \backslash \Pi)|>(\eta-\vartheta) n^{2} p
$$

(c) $\tau(B)>.04 \eta n^{3}$ and either $|G \cap B|>(1+.01 \eta) n^{2} p / 4$ or there is some $X \subseteq G \cap B$ of size at most $.005 \eta n^{2} p$ meeting all triangles of $G \cap B$.

Now - perhaps the main point - if $G_{0}$ is as in (38) (much more than we need here), then the number of possibilities for $F_{0}$ (once we have chosen $\left.G_{0}\right)$ is less than $2^{\vartheta n^{2} p}$. So for (41) it is enough to show that, for a given $F_{0}$ (again, with $B=B\left(F_{0}, G_{0}\right)$ satisfying (42)), each of the events (a)-(c) has probability at most $o\left(2^{-\vartheta n^{2} p}\right)$.

For (a), (b) and the event $\left\{|G \cap B|>(1+.01 \eta) n^{2} p / 4\right\}$ in (c) this is immediate from Theorem 2.1, which bounds the associated probabilities by expressions $\exp \left[-f(\eta) n^{2} p\right]$, with the $f(\eta)$ 's roughly $.01 \eta^{2} / 8, .005 \eta$ and $.0001 \eta^{2} / 8$ respectively. (It may be worth emphasizing that $B$ is determined by $F_{0}$; so e.g. in (a) we are interested in the probability that $G \cap B$ is large given that $B$ is small. The bound for (b) includes a factor $2^{n}$ for the number of possible $\Pi$ 's, which makes no difference since $n^{2} p=\omega(n)$.)

For the second alternative in (c), it is convenient to speak in terms of the hypergraph $\mathcal{H}$ whose vertices are the edges of $G^{\prime}:=G \cap B$ and whose edges are the triangles of $G^{\prime}$. Let $e_{1} \ldots e_{m}$ be the edges of $B$ and let $Y$ be the minimum size of a set of edges meeting all triangles of $G^{\prime}$. Since $Y$ is a Lipschitz function of the independent $\operatorname{Ber}(q)$ indicators $\mathbf{1}_{\left\{e_{i} \in G^{\prime}\right\}}$, Lemma 2.2 gives, for $0 \leq t \leq 2 m q$,

$$
\operatorname{Pr}(Y<\mathrm{E} Y-t)<\exp \left[-t^{2} /(4 m q)\right]
$$


On the other hand, we will show (assuming $\tau(B)>.04 \eta n^{3}$ as in (c))

$$
\mathrm{E} Y>.01 \eta n^{2} p .
$$

This will complete the proof, since (43) with $t=.005 \eta n^{2} p$ (now just using $m<n^{2} / 2$ and $q<p$ and noting that, for example, $\tau(B)>.04 \eta n^{3}$ implies $t<$ $2 m q$ ) then bounds the probability of an $X$ as in (c) by $\exp \left[-10^{-5} \eta^{2} n^{2} p\right]=$ $o\left(2^{-\vartheta n^{2} p}\right)$.

Proof of (44). We actually show the stronger

$$
\mathrm{E} \nu^{*}(\mathcal{H})>.01 \eta n^{2} p,
$$

where $\nu^{*}(\mathcal{H})$ is the fractional matching number of $\mathcal{H}$ (see e.g. [22]). To see this, say a triangle $T$ of $B$ is good if it is contained in $G^{\prime}$ and each of its edges lies in at most $1.9 n q^{2}$ triangles of $G^{\prime}$. Then for any $T \in T(B)$,

$$
\begin{aligned}
\operatorname{Pr}(T \text { is good }) & >q^{3}\left(1-3 \operatorname{Pr}\left(B\left(n, q^{2}\right)>1.9 n q^{2}\right)\right) \\
& >q^{3}\left(1-3 \exp \left[-n q^{2} / 4\right]\right) .
\end{aligned}
$$

Define a (random) weighting $w$ of the triangles of $G^{\prime}$ by

$$
w(T)=\left\{\begin{array}{cl}
\left(1.9 n q^{2}\right)^{-1} & \text { if } T \text { is good } \\
0 & \text { otherwise. }
\end{array}\right.
$$

Then $w$ is a fractional matching of $\mathcal{H}$, and we have (using (46))

$$
\mathrm{E} \nu^{*}(\mathcal{H}) \geq \tau(B)\left(1-3 \exp \left[-n q^{2} / 4\right]\right) q^{3}\left(1.9 n q^{2}\right)^{-1}>.01 \eta n^{2} p .
$$

\section{Coda}

Here we sketch an alternate proof of Theorem 1.2. The argument is similar to that in Sections 7 and 8 but seems worth including, as it is a little easier and shows that Theorem 1.4 and Lemma 3.2 were not really needed.

As in Section 7, we just need to show that for $p>1.2 \sqrt{\log n / n}$ and a fixed (small) $\eta>0$, it is unlikely that $\mathcal{T}^{\perp}(G)$ contains an $F$ for which (36) holds. We again fix some small $\vartheta$ (e.g. $\vartheta=0.1 \eta^{2}$ ) and choose $G$ by first choosing $G_{0} \sim G_{n, \vartheta p}$ and then adding edges of $K_{n} \backslash G_{0}$ with probability $(1-\vartheta) p /(1-\vartheta p)$. Of course we again have (38) w.h.p., and a discussion like that for (37) shows that w.h.p. any $F$ with (36) satisfies $|F \backslash \Pi|>0.1 n^{2} p$ for every cut $\Pi$. 
Given $G_{0}$ and $F_{0} \subseteq G_{0}$, set $A\left(G_{0}\right)=\left\{x y \in K_{n} \backslash G_{0}: N_{G_{0}}(x, y) \neq \emptyset\right\}$, $J=J\left(G_{0}\right)=K_{n} \backslash\left(G_{0} \cup A\left(G_{0}\right)\right)$, and

$$
B=B\left(F_{0}, G_{0}\right)=\left\{x y \in A\left(G_{0}\right): z \in N_{G_{0}}(x, y) \Rightarrow\left|\{x z, y z\} \cap F_{0}\right|=1\right\} .
$$

Then any $F \in \mathcal{T}^{\perp}(G)$ with $F \cap G_{0}=F_{0}$ satisfies $F \backslash\left(F_{0} \cup J\right)=G \cap B$.

Note also that w.h.p.

$$
|G \cap J|<o\left(n^{2} p\right)
$$

(e.g. by Theorem 2.1, using $\mathrm{E}|J|<n^{2}\left(1-(\vartheta p)^{2}\right)^{n-2}$ and Markov's Inequality to say that w.h.p. $\left.|J|=o\left(n^{2}\right)\right)$.

But if (38) and (47) hold and $F \in \mathcal{T}^{\perp}(G)$ satisfies (36), then there is an $F_{0} \subseteq G_{0}$ such that, with notation as above, we have one of:

(a) $|B|<(1-2 \eta) n^{2} / 4$ and $|G \cap B|>(1-\eta-2 \vartheta-o(1)) n^{2} p / 4$;

(b) there is a cut $\Pi$ with

$$
|B \backslash \Pi|<0.05 n^{2} \text { and }|G \cap(B \backslash \Pi)|>(0.1-\vartheta / 2-o(1)) n^{2} p ;
$$

(c) $\tau(B)>.004 n^{3}$ and $G \cap B$ is triangle-free.

Here we used (36) (to say $\left.|F|>(1-\eta) n^{2} p / 4\right)$, (38) and (47) for the second bound in $(\mathrm{a}) ;|G \cap(B \backslash \Pi)|=\left|F \backslash\left(\Pi \cup F_{0} \cup J\right)\right|$ together with $|F \backslash \Pi|>0.1 n^{2} p$, (38) and (47) for the second bound in (b); and Lemma 3.3 to say that failure of the conditions on $B$ in (a) and (b) implies the one in (c).

But, as in Section 8, (38) bounds the number of possibilities for $F_{0}$ (given $G_{0}$ ) by $2^{\vartheta n^{2} p}$, whereas, we assert, each of the events in (a)-(c) has probability $o\left(2^{-\vartheta n^{2} p}\right)$. For (a) and (b) this is again given by Theorem 2.1 (with (a) dictating the above choice of $\vartheta$ ). For (c) we may, for example, use an inequality of Janson ([13]; see also [14, Theorem 2.14]), as follows. Write $S$ for the set of (edge sets of) triangles of $B$; for $A \in S$, let $I_{A}$ be the indicator of $\{A \subseteq G\}$; and set $m=\tau(B)>.004 n^{3}$. Then $\mu:=\sum \mathrm{E} I_{A}=m p^{3}$ and $\bar{\Delta}:=\sum \sum_{A \cap B \neq \emptyset} \mathrm{E} I_{A} I_{B}<3 m n p^{5}+\mu$, and Janson's inequality bounds the probability that $G \cap B$ is triangle-free by (say)

$$
\exp \left[-\mu^{2} /(2 \bar{\Delta})\right]<\exp \left[-.0006 n^{2} p\right] .
$$

\section{References}

[1] N. Alon, personal communication.

[2] J. Balogh, personal communication. 
[3] J. Balogh, R. Morris and W. Samotij, Independent sets in hypergraphs, arXiv:1204.6530v1 [math.CO].

[4] D. Conlon and T. Gowers, Combinatorial theorems in sparse random sets, arXiv:1011.4310v1 [math.CO].

[5] B. DeMarco, Triangles in Random Graphs, thesis defense, Rutgers University, May 3, 2012.

[6] B. DeMarco and J. Kahn, Mantel's Theorem for random graphs (2012), http://arxiv.org/abs/1206.1016.

[7] R. Diestel, Graph Theory, Springer, New York, 2000. MR1743598

[8] P. Erdös, P. Frankl and V. Rödl, The asymptotic number of graphs not containing a fixed subgraph and a problem for hypergraphs having no exponent, Graphs Combin. 2 (1986), 112-121. MR0932119

[9] P. Erdős and A. Rényi, On random graphs I, Publ. Math. Debrecen 6 (1959), 290-297. MR0120167

[10] P. Frankl and V. Rödl, Large triangle-free subgraphs in graphs without $K_{4}$, Graphs and Combinatorics 2 (1986), 135-144. MR1884430

[11] A.W. Goodman, On sets of acquaintances and strangers at any party, Amer. Math. Mon. 66 (1959), 778-783. MR0107610

[12] P.E. Haxell, Y. Kohayakawa and T Łuczak, Turán's extremal problem in random graphs: forbidding odd cycles, Combinatorica 16 (1996), 107-122. MR1394514

[13] S. Janson, Poisson approximation for large deviations, Random Structures $\&$ Algorithms 1 (1990), 221-230. MR1138428

[14] S. Janson, T. Łuczak and A. Ruciński, Random Graphs, Wiley, New York, 2000. MR1782847

[15] J. Kahn, Asymptotically good list colorings, J. Combinatorial Th. (A) 73 (1996), 1-59. MR1367606

[16] M. Kahle, Topology of random clique complexes, Disc. Math. 309 (2009), 1658-1671. MR2510573

[17] M. Kahle, Sharp vanishing thresholds for cohomolgoy of random flag complexes, arXiv:1207.0149v1 [math.AT].

[18] M. Kahle, personal communication. 
[19] Y. Kohayakawa, Szemerédi's regularity lemma for sparse graphs, pp. 216-230 in Foundations of Computational Mathematics (Rio de Janeiro, 1997), Springer, Berlin, 1997. MR1661982

[20] Y. Kohayakawa, T. Euczak and V. Rödl, Arithmetic progressions of length three in subsets of a random set, Acta Arith. 75 (1996), 133163. MR1379396

[21] Y. Kohayakawa, T. Łuczak and V. Rödl, On $K^{4}$-free subgraphs of random graphs, Combinatorica 17 (1997), 173-213. MR1479298

[22] L. Lovász, Combinatorial Problems and Exercises, AMS Chelsea, Providence, 2007. MR2321240

[23] T. Łuczak, personal communication.

[24] R. Meshulam, The clique complex and hypergraph matching, Combinatorica 21 (2001), 89-94. MR1805715

[25] I. Ruzsa and E. Szemerédi, Triple systems with no six points carrying three triangles, pp. 939-945 in Combinatorics (Keszthely, 1976), Coll. Math. Soc. J. Bolyai 18, Vol. II. MR0519318

[26] W. Samotij, Stability results for discrete random structures, arXiv: $1111.6885 \mathrm{v} 1$ [math.CO].

[27] D. Saxton and A. Thomason, Hypergraph containers, arXiv: 1204.6595v1 [math.CO].

[28] M. Schacht, Extremal results for random discrete structures, www.math.uni-hamburg.de/home/schacht/preprints/extremal_rs.pdf.

[29] M. Simonovits, A method for solving extremal problems in graph theory, stability problems, pp. 279-319 in Theory of Graphs (Proc. Colloq., Tihany, 1966), Academic Press, New York, 1968. MR0233735

[30] E. Szemerédi, Regular partitions of graphs, pp. 399-401 in Problèmes combinatoires et théorie des graphes (Colloq. Internat. CNRS, Univ. Orsay, Orsay, 1976), Paris: Éditions du Centre National de la Recherche Scientifique (CNRS), 1978. MR0540024

[31] P. Turán, Eine Extremalaufgabe aus der Graphentheorie, Mat. Fiz Lapook 48 (1941), 436-452. MR0018405

B. Demarco

RUTGERS

USA

E-mail address: rvdemarco@gmail.com 


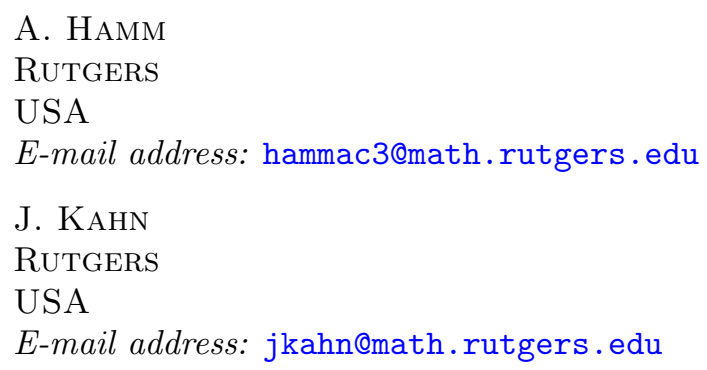

Received August 8, 2012 